\title{
Experiment on People's Selection of Route Landmarks from Different Types of Geospatial Pictures
}

\author{
Pyry Kettunen \\ Department of Geoinformatics and Cartography (FGI-GEOINFO), Finnish Geospatial Research Institute (FGI), National Land \\ Survey of Finland, pyry.kettunen@nls.fi
}

Keywords: geospatial picture, map, aerial image, route, landmark

\begin{abstract}
:
Different types of geospatial pictures, such as maps and aerial imagery, have varying potential to provide spatial knowledge for the viewers. Our earlier review has assessed different common types of geopictures with regard to their support for acquiring different types of spatial knowledge (Kettunen et al., 2012). The assessment was based on previous literature about geopicture experiments as well as analytical itemization of the information available on the pictures as a function of picture characteristics, namely vantage point, number of visible vertical features, and visual realism. In addition to the actual assessment, the review concluded that there is an important shortage of empirical evaluations on spatial knowledge acquisition from geopictures, which lag we are addressing with the experiment described here.
\end{abstract}

The aim of the experiment is to find out what kind of geospatial features, or landmarks (see Presson and Montello, 1988; Richter and Winter, 2014), people read from geopictures, does reading differ between pictures, and how does it differ. For the experimental task, we chose a common map activity of planning navigation for walking through an urban route. The stimuli are five different types of common geopictures with both aerial vertical and aerial oblique vantage points as well as with both abstract and photorealistic levels of visual realism (Figure 1). Five routes are to be planned in different areas of city of Helsinki so that a different geopicture type is shown for each route. The pictures are shown as corridors surrounding the shortest walking route.

The participants have been recruited from expert and non-expert social media groups and e-mail lists, being called to be over 18 years old. The background questionnaire has asked about their age, gender, possible visual disorders, and frequency of use for each type of map in the experiment.

The experimental design for measuring the effect of geopicture type was built randomized and between-participants so that each participant completes series of five pictures, each of different type, in randomized order, and from different geographical areas. Two rounds of five pictures are shown: first, to measure the actual selection of landmarks, and second, to investigate the level of recall of the selected landmarks later. The procedure of the experiment is the following (Figure 2): 1) Introduction, 2) Consent to participate, 3) Training trial of the landmark selection task (1 picture), 4) Testing trials of the landmark selection task (5 pictures), 5) Training trial of the selection recall task (1 picture), 6) Testing trials of the landmark selection task (5 pictures), 7) Background questionnaire, and 8) Thanks and feedback. The Start and End points of the routes are drawn on the pictures (Figure 1) and the participants are asked to plan the shortest route between the points along roads and paths, to point 3-7 features along the route in navigation route for being able to navigate the route, and to enter common names for the features after pointing each feature. Response times, pointed coordinates, and feature names are recorded in order to analyze the participants' answers.

The presentation at the ICC2019 conference will introduce preliminary results of the conducted experiment, reflect them on the previous studies on maps and routing and discuss their implications on the design of map contents from the viewpoint of route planning. The results will build empirical foundation about differences in reading of geospatial picture types, and for selecting appropriate features to be drawn on maps.

\section{References:}

Kettunen, P., Irvankoski, K., Krause, C. M., Sarjakoski, T. and L. T. Sarjakoski, 2012. Geospatial images in the acquisition of spatial knowledge for wayfinding. Journal of Spatial Information Science, 5:75-106. DOI: 10.5311/JOSIS.2012.5.85.

Presson, C. C. and D. R. Montello, 1988. Points of reference in spatial cognition: Stalking the elusive landmark. British Journal of Developmental Psychology, 6(4): 378-381. 10.1111/j.2044-835X.1988.tb01113.x.

Richter, K. F. and S. Winter, 2014. Landmarks. Springer. DOI: 10.1007/978-3-319-05732-3. 


\section{Acknowledgements:}

The author wishes to thank The Fulbright Program, Fulbright Finland Foundation, KAUTE Foundation and the Mobility Programme of the National Land Survey of Finland for the support of his research visit to the University of California, Santa Barbara (UCSB) in 2018, which made this research possible. At the UCSB, the author wishes to thank Prof. Daniel R. Montello for comments in planning this study and colleagues in the Center for Spatial Studies for help in the pilot study. Importantly, thanks go to every participant of the study.

a)

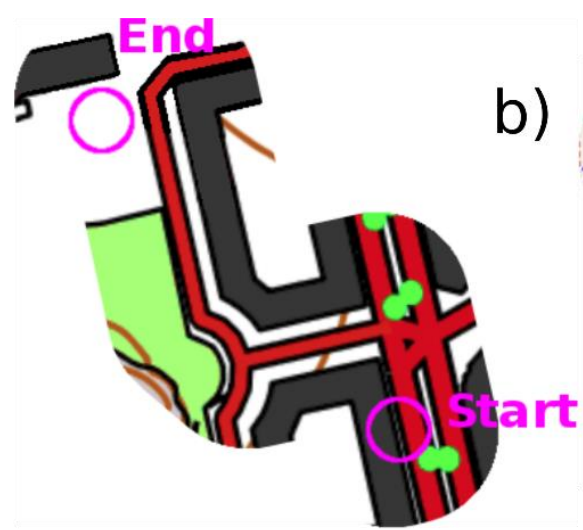

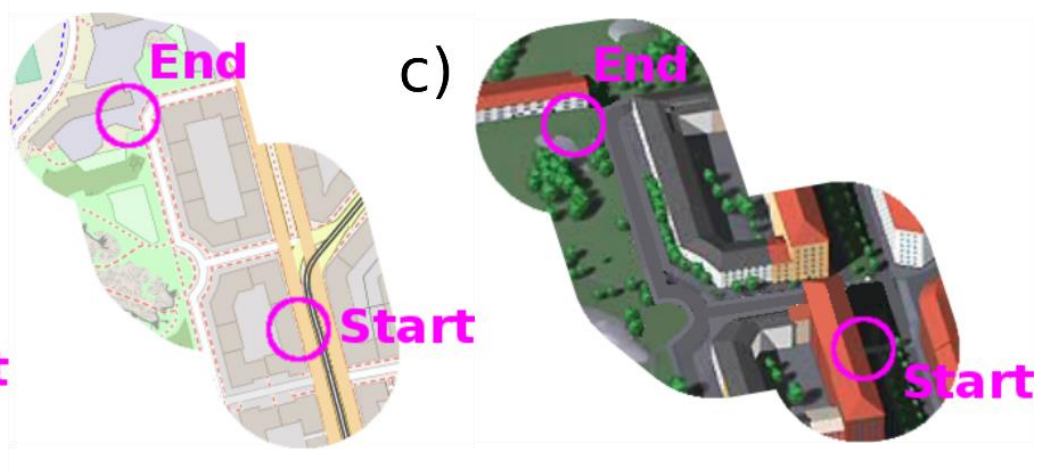

d)

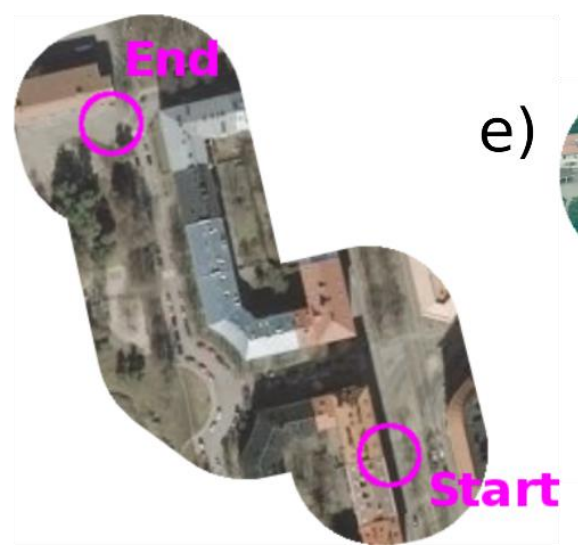

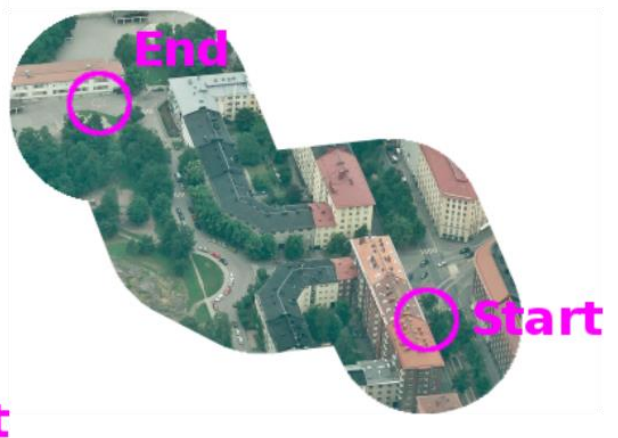

Figure 1. Geopicture stimuli in the experiment for one geographical area: a) Topographic map, b) Street map, c) 3D map, d) Aerial orthophoto, and e) Oblique aerial photo.

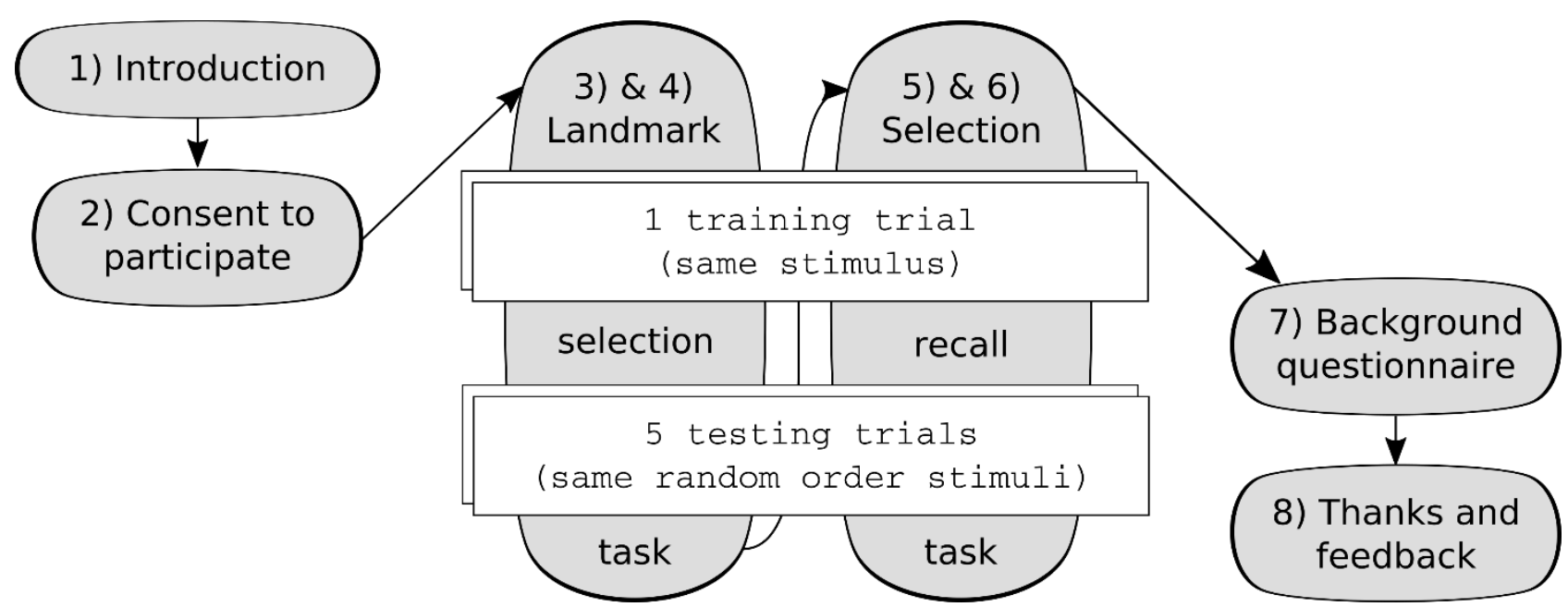

Figure 2. Procedure of the experiment. 